\title{
Soybean Disease Loss Estimates for the Southern United States, 1994 to 1996
}

\author{
Phillip W. Pratt, Oklahoma Cooperative Extension Service, Oklahoma State University, Muskogee 74401; and \\ J. Allen Wrather, Plant Science Unit, University of Missouri-Delta Center, Portageville 63873
}

Since 1974, soybean disease loss estimates for the southern United States have been published in the Southern Soybean Disease Workers (SSDW) Proceedings, summaries of which were published in 1978 (6), 1988 (2,3), 1993 (4), and 1995 (8). A summary of the results from 1974 to 1994 has also been published (7), and the 1994 results were published as part of the disease loss estimate for the top 10 soybean producing countries (9).

The loss estimates for 1994 to 1996 presented here were solicited from: Bill Gazaway, Alabama; Clifford Coker, Arkansas; Robert Mulrooney, Delaware; Tom Kucharek, Florida; Boyd Padgett, Georgia; Don Hershman, Kentucky; Ken Whitam, Louisiana; Arvydas Grybauskas, Maryland; Joe Fox, Mississippi; Allen Wrather, Missouri; Steve Koenning, North Carolina; Phil Pratt, Oklahoma; Charles Drye, South Carolina; Melvin Newman, Tennessee; Joseph Krausz, Texas; and Patrick Phipps, Virginia.

Various methods were used to identify the diseases of soybeans and to obtain disease loss estimates, with most estimates derived by more than one method. The methods used to assist in disease identification were field surveys, plant disease diagnostic clinic samples, questionnaires to Cooperative Extension staff, and private crop consultant reports. Methods used to obtain disease loss estimates were grower demonstrations conducted by the Cooperative Extension Service, private crop consultant reports, variety trials, foliar fungicide trials, and research plots. Yield losses were based on estimates of yield in the absence of disease.

The actual production figures for each state were supplied by the state crop reporting service. Dollars lost to disease were calculated by multiplying the estimated loss in metric tons by the average annual price of $\$ 222.67 / \mathrm{t}$ in 1994 (\$6.68/bu), \$208.33/t in $1995(\$ 6.25 / \mathrm{bu})$, and $\$ 263.33 / \mathrm{t}$ in 1996 (\$7.90/bu).

Corresponding author: P. W. Pratt

E-mail: pwpratt@ok.azalea.net

Accepted for publication 15 September 1997.

Publication no. D-1997-1103-01S

This article is in the public domain and not copyrightable. It may be freely reprinted with customary crediting of the source. The American Phytopathological Society, 1998.
Percentage of loss estimates from each state is specific for the causal organism or the common name of the disease. In 1995, the list of diseases was modified in order to obtain more specific information on the occurrence of diseases that in previous years had been placed together in general categories. For example, the category "root and lower stem rots" was separated into brown stem rot, Fusarium root rot, Sclerotinia stem rot, and southern blight. The category "foliar diseases (other)" was separated into aerial blight and frogeye leaf spot. The Diaporthe/Phomopsis complex was added to the list of diseases. Additional information on each disease can be found in the Compendium of Soybean Diseases (5).

During 1994, 16.18 million metric tons (594.45 million bushels) were harvested from 7.11 million hectares (17.57 million acres) in the southern states. Disease losses were estimated at $9.29 \%$, resulting in a production loss of 1.24 million metric tons (45.47 million bushels) valued at $\$ 303.74$ million $(\$ 6.68 / \mathrm{bu}$; Table 1$)$. The greatest percentage of loss occurred in Florida $(24.30 \%)$ and the least in Delaware $(4.10 \%)$. The greatest financial loss occurred in Missouri (\$67.33 million), followed by Arkansas ( $\$ 57.31$ million) and Louisiana (\$32.00 million).

During 1995, 12.43 million metric tons (456.70 million bushels) were harvested from 6.77 million hectares (16.73 million acres) in the southern states. Disease losses were estimated at $9.14 \%$, resulting in a production loss of 1.18 million metric tons (39.52 million bushels), valued at $\$ 247.03$ million $(\$ 6.25 / \mathrm{bu}$; Table 2). The greatest percentage of loss occurred in Alabama $(18.20 \%)$ and the least in Delaware $(2.50 \%)$. The greatest financial loss occurred in Missouri (\$76.29 million), followed by Arkansas (\$50.26 million) and Mississippi (\$27.87 million).

During 1996, 15.67 million metric tons (575.8 million bushels) were harvested from 7.05 million hectares (17.42 million acres) in the southern states. Disease losses were estimated at $8.67 \%$, resulting in a production loss of 1.58 million metric tons (58.22 million bushels) valued at $\$ 459.92$ million (\$7.90/bu; Table 3). The greatest percentage of loss occurred in Texas $(14.70 \%)$ and the least in Virginia (1.30\%). The greatest financial loss occurred in
Missouri (\$115.92 million), followed by Arkansas (\$99.89 million) and Mississippi (\$62.28 million).

The greatest economic loss over the 3year period was caused by soybean cyst nematode (\$275.38 million), followed by charcoal rot (\$159.22 million), Phytophthora root rot and stem rot $(\$ 84.43$ million), and root-knot and ectoparasitic nematodes ( $\$ 79.50$ million). Prior to 1995 , Phytophthora root and stem rot was not identified as an individual disease in the SSDW disease loss estimates. It was included in the broad category of "root and stem rots."

Diseases have been reported to cause significant loss in soybean production in the United States and in other major soybean-producing countries (1-9). Since 1977, soybean diseases have been reported as causing significant yield reduction in the southern soybean producing states (2-8). It is essential that University Research and Cooperative Extension continue their efforts to discover methods to control these diseases and to educate soybean producers concerning the best methods to prevent yield loss due to soybean diseases.

\section{LITERATURE CITED}

1. Doupnik, B., Jr. 1993. Soybean production and disease loss estimates for north central United States from 1989 to 1991. Plant Dis. 77:11701171.

2. Mulrooney, R. P. 1988. Soybean disease loss estimate for southern United States in 1985 and 1986. Plant Dis. 72:364-365.

3. Mulrooney, R. P. 1988. Soybean disease loss estimate for southern United States in 1987. Plant Dis. 72:915.

4. Sciumbato, G. L. 1993. Soybean disease loss estimate for the southern United States during 1988-1991. Plant Dis. 77:954-956.

5. Sinclair, J. B., and Backman, P. A., eds. 1989. Compendium of Soybean Diseases, 3rd ed. American Phytopathological Society, St. Paul, MN

6. Whitney, G. 1978. Southern states soybean disease loss estimates-1977. Plant Dis. Rep. 62:1078-1079.

7. Wrather, J. A., Chambers, A. Y., Fox, J. A., Moore, W. F., Sciumbato, G. L. 1995. Soybean disease loss estimates for the southern United States, 1974 to 1994. Plant Dis. 79:1076-1079.

8. Wrather, J. A., and Sciumbato, G. L. 1995. Soybean disease loss estimates for the southern United States during 1992-1993. Plant Dis. 79:84-85.

9. Wrather, J. A., Anderson, T. R., Arsyad, D. M., Gai, J., Ploper, L. D., Porta-Puglia, A., Ram, H. H., and Yorinori, J. T. 1997. Soybean disease loss estimates for the top 10 soybean producing countries in 1994. Plant Dis. 81:107-110. 
Table 1. Estimated percentage of loss to disease of soybean yields in 1994 in 16 southern states

\begin{tabular}{|c|c|c|c|c|c|c|c|c|c|c|c|c|c|c|c|c|c|}
\hline \multirow[b]{2}{*}{ Diseases } & \multicolumn{17}{|c|}{ Percent loss per state } \\
\hline & $\mathbf{A L}$ & $\mathbf{A R}$ & DE & FL & GA & KY & LA & MD & MS & MO & $\mathrm{NC}$ & OK & SC & $\mathbf{T N}$ & $\mathbf{T X}$ & VA & $\operatorname{Avg}^{\mathrm{a}}$ \\
\hline Anthracnose & 1.00 & 0.40 & $\mathrm{TR}^{\mathrm{b}}$ & 2.00 & 0.25 & 0.50 & 0.50 & TR & 0.78 &..$^{b}$ & 0.40 & 1.50 & 1.50 & 1.00 & 3.00 & $\ldots$ & 0.80 \\
\hline Bacterial diseases & 0.10 & 0.10 & & $\ldots$ & $\ldots$ & $\mathrm{TR}$ & $\mathrm{TR}$ & $\ldots$ & $\mathrm{TR}$ & & 0.05 & $\mathrm{TR}$ & 0.05 & $\mathrm{TR}$ & 0.10 & $\ldots$ & 0.03 \\
\hline Brown spot & 0.50 & & $\mathrm{TR}$ & $\ldots$ & $\ldots$ & 0.50 & TR & $\ldots$ & 0.06 & & 0.05 & TR & 0.15 & 1.00 & 0.10 & $\mathrm{TR}$ & 0.15 \\
\hline Charcoal rot & 0.10 & 1.00 & & 0.10 & & 0.05 & 1.00 & $\ldots$ & 2.60 & 2.00 & 0.05 & 2.00 & 0.07 & 0.50 & 1.00 & $\ldots$ & 0.65 \\
\hline $\begin{array}{l}\text { Diaporthe pod \& stem } \\
\text { blight }\end{array}$ & 0.60 & 0.10 & TR & 2.00 & 0.75 & 1.20 & 2.00 & 0.50 & 0.90 & $\ldots$ & 0.04 & 1.00 & 1.00 & $\ldots$ & 0.50 & 0.10 & 0.67 \\
\hline Downy mildew & 0.10 & 0.10 & $\mathrm{TR}$ & 0.10 & $\ldots$ & $\mathrm{TR}$ & $\mathrm{TR}$ & $\ldots$ & $\mathrm{TR}$ & $\ldots$ & 0.02 & TR & 0.10 & 0.01 & $\mathrm{TR}$ & $\ldots$ & 0.03 \\
\hline Foliar diseases (other) & 0.40 & 0.50 & $\ldots$ & $\ldots$ & 2.00 & $\ldots$ & 1.00 & $\ldots$ & 0.33 & $\ldots$ & 0.30 & 0.50 & 0.50 & 0.01 & 0.40 & $\ldots$ & 0.37 \\
\hline Other diseases $^{\mathrm{c}}$ & $\ldots$ & & & $\ldots$ & & $\ldots$ & 3.00 & $\ldots$ & $\ldots$ & $\ldots$ & $\ldots$ & & $\ldots$ & 0.01 & $\ldots$ & 0.20 & 0.20 \\
\hline Purple stain & 0.10 & 0.20 & $\mathrm{TR}$ & 0.10 & 0.75 & 0.02 & 2.00 & TR & 0.53 & & 0.02 & 0.50 & 0.70 & & 1.00 & 0.10 & 0.38 \\
\hline Root \& stem rots & 0.10 & 0.25 & $\mathrm{TR}$ & 10.00 & 0.25 & 0.05 & 1.00 & $\ldots$ & 1.60 & 1.00 & 0.40 & 0.20 & 1.10 & 0.50 & 0.70 & 0.10 & 1.08 \\
\hline $\begin{array}{l}\text { Root-knot \& } \\
\text { other nematodes }\end{array}$ & 1.00 & 0.20 & 1.00 & 7.00 & 3.50 & $\ldots$ & 2.00 & 0.50 & 0.02 & & 2.50 & TR & 7.00 & 0.10 & 0.40 & 1.00 & 1.64 \\
\hline Seedling diseases & 0.20 & 1.50 & $\ldots$ & 2.00 & 0.50 & 0.20 & $\mathrm{TR}$ & $\ldots$ & 0.45 & $\ldots$ & 0.05 & 0.20 & 0.10 & 1.50 & 0.50 & 0.20 & 0.46 \\
\hline Soybean cyst nematode & 1.50 & 0.80 & 3.00 & 1.00 & 3.00 & 2.50 & 1.00 & 7.00 & 0.86 & 3.00 & 4.50 & 0.75 & 3.00 & 2.00 & 0.10 & 3.40 & 2.34 \\
\hline Stem canker & 0.10 & 0.75 & $\ldots$ & $\ldots$ & $\ldots$ & 0.05 & 0.50 & $\ldots$ & $\mathrm{TR}$ & $\ldots$ & $\ldots$ & $\ldots$ & 0.01 & 0.02 & 0.50 & $\ldots$ & 0.12 \\
\hline Sudden death syndrome & 0.40 & 0.75 & & $\ldots$ & & 0.05 & TR & $\ldots$ & TR & $\ldots$ & $\ldots$ & & $\ldots$ & 0.50 & 0.10 & $\ldots$ & 0.11 \\
\hline Virus diseases & 0.20 & 0.60 & 0.10 & $\ldots$ & 0.50 & TR & 1.00 & $\ldots$ & TR & & 0.40 & TR & 1.00 & TR & 0.30 & 0.10 & 0.26 \\
\hline Total percent loss ${ }^{\mathrm{a}}$ & 6.40 & 7.25 & 4.10 & 24.30 & 11.50 & 5.12 & 15.00 & 8.00 & 8.13 & 6.00 & 8.78 & 6.65 & 16.28 & 7.15 & 8.70 & 5.20 & 9.29 \\
\hline Yield $(\mathrm{t} / \mathrm{ha})^{\mathrm{a}, \mathrm{d}}$ & 1.95 & 2.49 & 2.42 & 1.61 & 2.02 & 2.49 & 1.95 & 2.62 & 1.95 & 2.49 & 2.08 & 1.61 & 1.68 & 2.29 & 2.08 & 2.22 & 2.12 \\
\hline Total hectares $\left(\times 10^{6}\right)^{\mathrm{a}}$ & 0.12 & 1.30 & 0.09 & 0.02 & 0.20 & 0.45 & 0.45 & 0.22 & 0.81 & 1.84 & 0.55 & 0.11 & 0.25 & 0.43 & 0.08 & 0.21 & 7.11 \\
\hline Yield loss $\left(\mathrm{t} \times 10^{5}\right)^{\mathrm{a}, \mathrm{d}}$ & 0.15 & 2.33 & 0.09 & 0.06 & 0.45 & 0.58 & 1.30 & 0.47 & 1.28 & 2.74 & 1.00 & 0.14 & 0.69 & 0.69 & 0.15 & 0.24 & 12.37 \\
\hline Dollar loss $\left(\times 10^{6}\right)^{\mathrm{a}, \mathrm{e}}$ & 3.74 & 57.31 & 2.20 & 1.54 & 11.02 & 14.16 & 32.00 & 11.49 & 31.53 & 67.33 & 24.65 & 3.34 & 16.83 & 17.03 & 3.61 & 5.95 & 303.74 \\
\hline
\end{tabular}

${ }^{a}$ Rounding errors present.

b $\mathrm{TR}=$ trace

c Other diseases not identified in LA, TN, and VA.

${ }^{\mathrm{d}}$ The yield loss is based on the percentage of loss of what yield would have been had no diseases occurred.

${ }^{\text {e }}$ Dollar loss $=$ estimated loss $\times \$ 245.49 / \mathrm{t}$.

Table 2. Estimated percentage of loss to disease of soybean yields in 1995 in 16 southern states

\begin{tabular}{|c|c|c|c|c|c|c|c|c|c|c|c|c|c|c|c|c|c|}
\hline \multirow[b]{2}{*}{ Diseases } & \multicolumn{17}{|c|}{ Percent loss per state } \\
\hline & $\mathbf{A L}$ & $\mathbf{A R}$ & DE & FL & GA & KY & LA & MD & MS & MO & $\mathrm{NC}$ & OK & SC & $\mathbf{T N}$ & $\mathbf{T X}$ & VA & Avg $^{\mathrm{a}}$ \\
\hline Anthracnose & 2.00 & 0.51 & $\ldots{ }^{b}$ & 2.00 & 0.01 & 0.50 & 0.50 & 0.20 & 0.30 & $\ldots$ & 0.80 & 0.30 & 2.00 & 0.50 & 3.00 & $\mathrm{TR}^{\mathrm{b}}$ & 0.79 \\
\hline Bacterial diseases & 0.50 & 0.01 & $\ldots$ & $\ldots$ & $\mathrm{TR}$ & TR & TR & $\ldots$ & TR & 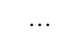 & 0.05 & 1.00 & 0.05 & TR & 0.10 & $\ldots$ & 0.11 \\
\hline Brown spot & 0.20 & 0.05 & $\mathrm{TR}$ & $\ldots$ & $\ldots$ & 0.50 & TR & 0.10 & 0.10 & 0.10 & 0.10 & 0.30 & 0.15 & 0.50 & 0.20 & $\ldots$ & 0.14 \\
\hline Brown stem rot & $\ldots$ & $\ldots$ & $\ldots$ & $\ldots$ & $\ldots$ & TR & $\ldots$ & $\ldots$ & $\ldots$ & 0.10 & $\ldots$ & $\ldots$ & $\ldots$ & $\ldots$ & 0.10 & $\ldots$ & 0.01 \\
\hline Charcoal rot & & 1.9 & 0.50 & 0.10 & $\ldots$ & 0.10 & 2.00 & 0.05 & 5.80 & 1.00 & 0.05 & 2.00 & 0.01 & 0.70 & 1.00 & TR & 0.95 \\
\hline Diaporthe/Phomopsis & 2.00 & 0.01 & $\mathrm{TR}$ & 2.00 & 0.25 & 0.20 & 1.00 & $\ldots$ & $\ldots$ & $\ldots$ & 4.30 & 1.50 & $\ldots$ & & 1.00 & $\ldots$ & 0.77 \\
\hline Downy mildew & 1.00 & 0.01 & TR & TR & $\ldots$ & TR & TR & $\ldots$ & TR & $\ldots$ & 0.06 & 0.50 & 0.10 & 0.01 & TR & $\ldots$ & 0.11 \\
\hline Frogeye leaf spot & 1.00 & 0.42 & $\ldots$ & TR & $\mathrm{TR}$ & TR & 0.25 & $\ldots$ & 0.30 & $\ldots$ & 0.30 & 0.20 & 0.05 & 0.02 & 1.00 & $\ldots$ & 0.22 \\
\hline Fusarium wilt \& rot & $\ldots$ & 0.02 & $\ldots$ & $\ldots$ & 0.25 & TR & TR & $\ldots$ & $\ldots$ & 1.00 & $\ldots$ & $\ldots$ & 0.01 & $\ldots$ & $\ldots$ & $\ldots$ & 0.08 \\
\hline Other diseases ${ }^{\mathrm{c}}$ & $\ldots$ & $\ldots$ & $\ldots$ & $\ldots$ & $\ldots$ & $\ldots$ & $\ldots$ & $\ldots$ & $\ldots$ & $\ldots$ & TR & $\ldots$ & $\ldots$ & 0.01 & 0.30 & $\mathrm{TR}$ & 0.02 \\
\hline Phytophthora rot & & 0.03 & $\ldots$ & $\ldots$ & $\ldots$ & 0.05 & 0.50 & $\ldots$ & 0.20 & 2.00 & 0.30 & 0.10 & 0.01 & 0.01 & & $\ldots$ & 0.20 \\
\hline Pod \& stem blight & 3.00 & 0.50 & TR & $\ldots$ & 0.50 & 0.20 & 1.00 & 0.10 & 0.50 & $\ldots$ & TR & 1.50 & 1.00 & $\ldots$ & 0.30 & 0.10 & 0.54 \\
\hline Purple stain & & 0.30 & $\ldots$ & 0.20 & 0.25 & 0.10 & 1.00 & 0.05 & 0.50 & $\ldots$ & 0.20 & 1.00 & 1.00 & $\ldots$ & 2.50 & $\mathrm{TR}$ & 0.44 \\
\hline $\begin{array}{l}\text { Rhizoctonia aerial } \\
\text { blight }\end{array}$ & 0.30 & 0.06 & $\ldots$ & 0.10 & $\ldots$ & $\ldots$ & 0.50 & $\ldots$ & $\mathrm{TR}$ & $\cdots$ & $\ldots$ & $\ldots$ & 0.01 & $\ldots$ & 0.30 & $\ldots$ & 0.08 \\
\hline $\begin{array}{l}\text { Root-knot \& other } \\
\text { nematodes }\end{array}$ & 2.50 & 0.80 & $\mathrm{TR}$ & 3.00 & 3.50 & $\ldots$ & 2.00 & 1.00 & 0.10 & 0.50 & 2.00 & TR & 7.00 & 0.10 & 0.30 & 0.50 & 1.46 \\
\hline Sclerotina stem rot & & $\ldots$ & $\ldots$ & & $\ldots$ & $\ldots$ & TR & $\ldots$ & $\ldots$ & & & $\ldots$ & $\ldots$ & & 0.10 & $\ldots$ & 0.01 \\
\hline Seedling diseases & 0.50 & 1.11 & $\ldots$ & 1.00 & 0.50 & 0.30 & TR & $\ldots$ & 0.30 & 0.50 & TR & 0.30 & 0.10 & 1.00 & 0.50 & 0.10 & 0.39 \\
\hline Southern blight & 0.20 & $\ldots$ & $\ldots$ & 0.10 & 0.01 & TR & & $\ldots$ & TR & & TR & $\mathrm{TR}$ & 1.00 & & $\ldots$ & $\mathrm{TR}$ & 0.08 \\
\hline $\begin{array}{l}\text { Soybean cyst } \\
\text { nematode }\end{array}$ & 3.00 & 3.10 & 2.00 & 1.00 & 3.00 & 3.50 & 0.50 & 5.00 & 0.10 & 2.50 & 5.00 & 0.70 & 3.00 & 1.50 & 0.10 & 2.50 & 2.28 \\
\hline Stem canker & 1.00 & 0.27 & $\ldots$ & $\ldots$ & $\ldots$ & 0.02 & TR & $\ldots$ & 0.30 & $\ldots$ & $\ldots$ & $\ldots$ & 0.01 & 0.01 & 0.50 & $\ldots$ & 0.13 \\
\hline $\begin{array}{l}\text { Sudden death } \\
\text { syndrome }\end{array}$ & 1.00 & 0.27 & $\ldots$ & $\ldots$ & $\ldots$ & 0.08 & $\ldots$ & $\ldots$ & $\mathrm{TR}$ & 0.50 & $\ldots$ & $\ldots$ & $\ldots$ & 0.60 & 0.10 & $\ldots$ & 0.16 \\
\hline Virus & $\ldots$ & 0.03 & $\mathrm{TR}$ & $\ldots$ & 0.25 & 0.03 & 0.50 & $\ldots$ & TR & $\ldots$ & 0.40 & 0.10 & 1.00 & TR & 0.30 & 0.10 & 0.17 \\
\hline Total percent loss ${ }^{\mathrm{a}}$ & 18.20 & 9.40 & 2.53 & 9.51 & 8.53 & 5.62 & 9.79 & 6.50 & 8.53 & 8.20 & 13.58 & 9.51 & 16.50 & 4.97 & 11.71 & 3.33 & 9.14 \\
\hline Yield (t/ha) $)^{\mathrm{a}, \mathrm{d}}$ & 1.55 & 1.68 & 1.34 & 2.15 & 1.61 & 2.55 & 1.68 & 1.68 & 1.61 & 2.02 & 1.55 & 1.68 & 1.61 & 2.08 & 1.68 & 1.81 & 1.77 \\
\hline Total hectares $\left(\times 10^{6}\right)^{\mathrm{a}}$ & 0.09 & 1.26 & 0.09 & 0.01 & 0.13 & 0.46 & 0.40 & 0.22 & 0.81 & 1.84 & 0.43 & 0.10 & 0.21 & 0.43 & 0.10 & 0.20 & 6.77 \\
\hline Yield loss $\left(\mathrm{t} \times 10^{5}\right)^{\mathrm{a}, \mathrm{d}}$ & 0.31 & 2.19 & 0.03 & 0.03 & 0.19 & 0.69 & 0.79 & 0.27 & 1.29 & 3.32 & 1.03 & 0.18 & 0.68 & 0.46 & 0.22 & 0.12 & 11.81 \\
\hline Dollar loss $\left(\times 10^{6}\right)^{\mathrm{a}, \mathrm{e}}$ & 7.04 & 50.26 & 0.74 & 0.59 & 4.48 & 15.96 & 18.05 & 6.26 & 29.66 & 76.21 & 23.71 & 4.11 & 15.71 & 10.64 & 4.98 & 2.87 & 271.64 \\
\hline
\end{tabular}


Table 3. Estimated percentage of loss to disease of soybean yields in 1996 in 16 southern states

\begin{tabular}{|c|c|c|c|c|c|c|c|c|c|c|c|c|c|c|c|c|c|}
\hline \multirow[b]{2}{*}{ Diseases } & \multicolumn{17}{|c|}{ Percent loss per state } \\
\hline & $\mathbf{A L}$ & $\mathbf{A R}$ & DE & FL & GA & KY & $\mathbf{L A}$ & MD & MS & MO & NC & OK & $\mathrm{SC}$ & $\mathbf{T N}$ & TX & VA & Avg $^{\mathbf{a}}$ \\
\hline Anthracnose & 1.60 & 0.58 & $\ldots{ }^{b}$ & 2.00 & 0.20 & 1.20 & 0.05 & $\ldots$ & 0.60 & $\ldots$ & 0.40 & 0.40 & 1.00 & 0.50 & 2.50 & $\ldots$ & 0.69 \\
\hline $\begin{array}{r}\text { Bacterial } \\
\text { diseases }\end{array}$ & $\ldots$ & 0.03 & $\ldots$ & $\cdots$ & $\mathrm{TR}^{\mathrm{b}}$ & 0.02 & TR & $\ldots$ & TR & $\ldots$ & 0.05 & 0.20 & 0.05 & TR & 0.10 & $\ldots$ & 0.03 \\
\hline Brown spot & 0.60 & 0.04 & 0.5 & $\ldots$ & $\ldots$ & 0.20 & 0.20 & 0.10 & 0.20 & $\ldots$ & 0.05 & TR & 0.10 & 0.50 & 0.30 & $\ldots$ & 0.17 \\
\hline Brown stem rot & $\ldots$ & $\ldots$ & $\ldots$ & $\ldots$ & $\ldots$ & $\ldots$ & $\ldots$ & $\ldots$ & $\ldots$ & $\ldots$ & $\ldots$ & $\ldots$ & $\ldots$ & $\ldots$ & 0.10 & $\ldots$ & 0.01 \\
\hline Charcoal rot & $\ldots$ & 1.95 & $\ldots$ & 0.50 & $\ldots$ & 0.10 & 1.00 & $\ldots$ & 4.80 & 1.00 & 0.05 & 0.80 & 0.10 & 1.00 & 2.00 & $\ldots$ & 0.83 \\
\hline $\begin{array}{l}\text { Diaporthe/ } \\
\text { Phomopsis }\end{array}$ & 0.30 & 0.01 & $\cdots$ & 3.00 & 0.25 & 0.70 & 1.00 & 0.05 & $\ldots$ & $\ldots$ & 0.08 & 1.50 & TR & $\ldots$ & 1.00 & $\ldots$ & 0.49 \\
\hline Downy mildew & 0.20 & 0.01 & $\ldots$ & TR & $\ldots$ & 0.02 & TR & 0.05 & TR & $\ldots$ & 0.08 & 0.20 & 0.05 & 0.01 & $\ldots$ & $\ldots$ & 0.03 \\
\hline $\begin{array}{l}\text { Frogeye } \\
\text { leaf spot }\end{array}$ & 0.30 & 0.21 & $\cdots$ & 0.50 & TR & 0.05 & 0.50 & $\cdots$ & 0.20 & $\cdots$ & 0.10 & TR & 0.10 & 0.03 & 1.00 & $\cdots$ & 0.19 \\
\hline $\begin{array}{l}\text { Fusarium } \\
\text { wilt \& rot }\end{array}$ & 0.10 & 0.03 & $\ldots$ & $\cdots$ & 0.25 & 0.01 & $\ldots$ & $\ldots$ & $\cdots$ & $\cdots$ & $\cdots$ & $\cdots$ & 0.01 & $\ldots$ & 0.50 & $\ldots$ & 0.06 \\
\hline Other diseases $^{c}$ & $\ldots$ & $\ldots$ & $\ldots$ & $\ldots$ & TR & $\ldots$ & $\ldots$ & $\ldots$ & $\ldots$ & $\ldots$ & 5.00 & $\ldots$ & 0.60 & 0.20 & 1.30 & $\ldots$ & 0.44 \\
\hline $\begin{array}{l}\text { Phytophthora } \\
\text { rot }\end{array}$ & $\ldots$ & 0.10 & $\ldots$ & $\ldots$ & $\ldots$ & 0.02 & 0.50 & $\ldots$ & 1.10 & 2.00 & 0.40 & 0.01 & 0.01 & 0.01 & 0.10 & $\ldots$ & 0.27 \\
\hline $\begin{array}{l}\text { Pod \& stem } \\
\text { blight }\end{array}$ & 0.40 & 1.00 & $\ldots$ & 3.00 & 0.75 & 0.70 & 2.00 & 0.10 & 0.50 & $\cdots$ & 0.01 & 1.50 & 0.95 & $\ldots$ & 0.30 & $\mathrm{TR}$ & 0.70 \\
\hline Purple stain & 0.30 & 0.50 & $\cdots$ & 0.20 & 0.25 & 0.03 & 1.00 & 0.10 & 1.00 & $\cdots$ & 0.20 & 0.40 & 0.25 & $\cdots$ & 3.00 & $\mathrm{TR}$ & 0.45 \\
\hline $\begin{array}{l}\text { Rhizoctonia } \\
\text { aerial blight }\end{array}$ & $\ldots$ & 0.06 & $\cdots$ & 0.10 & $\cdots$ & $\ldots$ & 4.00 & $\ldots$ & 0.10 & $\cdots$ & $\cdots$ & $\cdots$ & 0.01 & $\ldots$ & 0.30 & $\ldots$ & 0.29 \\
\hline $\begin{array}{l}\text { Root-knot \& } \\
\text { other } \\
\text { nematodes }\end{array}$ & 1.00 & 0.45 & $\ldots$ & 2.50 & 3.50 & $\ldots$ & 1.50 & 0.05 & 0.20 & $\ldots$ & 1.50 & $\mathrm{TR}$ & 5.00 & 0.11 & 0.30 & 0.20 & 1.02 \\
\hline $\begin{array}{l}\text { Sclerotina } \\
\text { stem rot }\end{array}$ & $\ldots$ & $\ldots$ & $\ldots$ & $\cdots$ & $\ldots$ & $\ldots$ & $\ldots$ & $\ldots$ & $\ldots$ & $\ldots$ & $\ldots$ & $\ldots$ & $\ldots$ & $\ldots$ & 0.10 & $\ldots$ & 0.01 \\
\hline Southern blight & $\ldots$ & 0.005 & $\ldots$ & 0.10 & 0.01 & $\ldots$ & $\ldots$ & $\ldots$ & TR & $\ldots$ & 0.03 & 0.01 & 1.00 & $\ldots$ & 0.30 & $\ldots$ & 0.09 \\
\hline $\begin{array}{l}\text { Seedling } \\
\text { diseases }\end{array}$ & 0.10 & 1.85 & $\cdots$ & 1.00 & 0.50 & 0.20 & TR & $\cdots$ & 0.50 & 1.00 & 0.03 & 0.20 & 0.10 & 1.20 & 0.50 & $\cdots$ & 0.44 \\
\hline $\begin{array}{l}\text { Soybean cyst } \\
\text { nematode }\end{array}$ & 1.50 & 2.55 & 2.00 & 0.10 & 3.00 & 4.30 & 1.00 & 2.00 & 0.20 & 4.50 & 4.00 & 0.70 & 3.00 & 1.50 & 0.10 & 1.00 & 1.97 \\
\hline Stem canker & 0.10 & 0.10 & $\cdots$ & $\ldots$ & $\ldots$ & 0.10 & TR & ... & 0.30 & $\ldots$ & $\ldots$ & $\cdots$ & $\ldots$ & 0.05 & 0.50 & $\ldots$ & 0.07 \\
\hline $\begin{array}{l}\text { Sudden death } \\
\text { syndrome }\end{array}$ & 0.40 & 0.22 & $\ldots$ & $\cdots$ & $\cdots$ & 0.10 & $\ldots$ & $\ldots$ & 0.10 & $\ldots$ & $\cdots$ & $\cdots$ & $\cdots$ & 0.60 & 0.10 & $\ldots$ & 0.10 \\
\hline Virus & $\ldots$ & 0.20 & 0.10 & $\ldots$ & 0.25 & 0.05 & 0.50 & TR & 1.50 & $\ldots$ & 0.60 & 0.05 & 1.50 & TR & 0.30 & 0.10 & 0.32 \\
\hline $\begin{array}{l}\text { Total } \\
\text { percent loss }{ }^{\mathrm{a}}\end{array}$ & 6.90 & 9.90 & 2.60 & 13.00 & 8.96 & 7.80 & 13.25 & 2.45 & 11.30 & 8.50 & 12.58 & 5.97 & 13.83 & 5.71 & 14.70 & 1.30 & 8.67 \\
\hline Yield (t/ha) $)^{\mathrm{a}, \mathrm{d}}$ & 2.08 & 2.15 & 2.15 & 2.02 & 1.81 & 2.55 & 2.22 & 2.29 & 2.08 & 2.62 & 1.95 & 1.61 & 1.75 & 2.15 & 1.68 & 2.35 & 2.09 \\
\hline $\begin{array}{l}\text { Total hectares } \\
\left(\times 10^{6}\right)^{\mathrm{a}}\end{array}$ & 0.13 & 1.54 & 0.09 & 0.01 & 0.16 & 0.48 & 0.41 & 0.19 & 0.81 & 1.64 & 0.49 & 0.12 & 0.22 & 0.47 & 0.11 & 0.19 & 7.05 \\
\hline $\begin{array}{l}\text { Yield loss } \\
\left(\mathrm{t} \times 10^{5}\right)^{\mathrm{a}, \mathrm{d}}\end{array}$ & 0.20 & 3.44 & 0.05 & 0.04 & 0.29 & 1.03 & 1.41 & 0.11 & 2.15 & 3.99 & 1.36 & 0.11 & 0.61 & 0.61 & 0.38 & 0.06 & 15.84 \\
\hline $\begin{array}{r}\text { Dollar loss } \\
\left(\times 10^{6}\right)^{\mathrm{a}, \mathrm{e}}\end{array}$ & 5.72 & 99.89 & 1.45 & 1.17 & 8.40 & 29.96 & 40.83 & 3.24 & 62.28 & 115.92 & 39.56 & 3.31 & 17.81 & 17.61 & 11.03 & 1.75 & 459.92 \\
\hline
\end{tabular}

a Rounding errors present.

b $\mathrm{TR}=$ trace.

c Other diseases include loss due to ozone damage and black root rot (NC), and Cylindrocladium black root rot (SC). Other diseases not identified in GA, $\mathrm{TN}$, and TX.

d The yield loss is based on the percentage of loss of what yield would have been had no diseases occurred.

e Dollar loss $=$ estimated loss $\times \$ 290.33 / \mathrm{t}$. 